PACIFIC JOURNAL OF MATHEMATICS

Vol. 178, No. 1, 1997

\title{
REGULARITY OF CONVOLUTION OPERATORS
}

\author{
H.D. FEGAN
}

In this paper we prove that any operator which is given by convolution with a suitable distribution on a compact semisimple Lie group is of type $\left(\frac{1}{2}, \frac{1}{2}\right)$. Our main result is:

Theorem 1.1. If $K$ is an operator defined by convolution, so $K f=$ $k * f$, then, for suitable distributions $k$, the operator $K$ has the type $\left(\frac{1}{2}, \frac{1}{2}\right)$.

\section{Introduction.}

This question first arose for us in [2] where we gave an example in answer to a question raised by Gilkey [3]. Key to this example was a convolution operator. In the special case of interest in [2] this operator could be identified with a Szegö projector and so recognized as being of type $\left(\frac{1}{2}, \frac{1}{2}\right)$. In this work we are addressing the more general question. It is clear from [8] that a convolution operator has type $(\rho, 1-\rho)$ providing $k$ satisfies a suitable growth condition involving $\rho$, in our case $\rho=\frac{1}{2}$. While this is plausible there is no readily available proof of such an a priori growth estimate.

It is not surprising that it is difficult to obtain these growth estimates. We are essentially characterizing the regularity of an operator in terms of its Fourier coefficients. Even for functions on Euclidean space this is well known to be difficult. For example if $f=\Sigma c_{k} e^{i k x}$ then $\Sigma\left|c_{k}\right|^{2}<\infty$ is sufficient to establish $f \in L^{p}$ only for $p=2$. For this and other examples of the difficulty of detecting regularity from Fourier coefficients see [5].

What does it mean for an operator to have type $\left(\frac{1}{2}, \frac{1}{2}\right)$ ? Given a classical elliptical pseudo differential operator one important question is that of inverting the operators. However, the inverse need not be a classical pseudo differential operator. In [4] Hormander introduced a class of operators, those of type $\left(\frac{1}{2}, \frac{1}{2}\right)$, which included the inverses of the classical operators. On $\mathbb{R}^{n}$ these operators are given by a symbol $q$;

$$
Q f(x)=(2 \pi)^{-n} \int e^{i x \cdot \xi} q(x, \xi) \hat{f}(\xi) d \xi
$$


where $\hat{f}$ is the Fourier transform of $f$. This operator is of type $(\rho, \delta)$ if $q$ satisfies the growth conditions

$$
\left|D_{x}^{\beta} D_{\xi}^{\alpha} q(x, \xi)\right|<C_{\alpha, \beta}\left(1+|\xi|^{2}\right)^{(m-\rho|\alpha|+\delta|\beta|) / 2},
$$

for all multi-indices $\alpha$ and $\beta$. The classical pseudo differential operators are those of type $(1,0)$. By using local coordinates these classes of operators can be defined on compact manifolds.

In Section 2 we describe precisely what is mean by a suitable distribution. Briefly on a compact semisimple Lie group $G$ the set of irreducible representations, $\pi_{\lambda}: G \rightarrow$ Aut $E_{\lambda}$, is indexed by the highest weights $\lambda$. If $\left\{v_{i}^{\lambda}\right\}$ is a basis for $E_{\lambda}$ then the matrix coefficients are $u_{\lambda}^{i j}(x)=\left\langle\pi_{\lambda}(x) v_{i}^{\lambda}, v_{j}^{\lambda}\right\rangle$. A distribution can then be expressed as a sum of matrix coefficients

$$
k(x)=\sum_{\lambda, i, j} a_{\lambda}^{i j} u_{\lambda}^{i j}
$$

We define our classes of distributions, $\mathcal{D}^{h}$, by requiring that $a_{\lambda}^{i j}$ have polynomial growth:

$$
\left|a_{\lambda}^{i j}\right|<C|\lambda|^{r} \quad \text { as }|\lambda| \rightarrow \infty
$$

In (1.4) $r$ is related to $h, r=h-\ell-m$, with $\ell=\operatorname{rank} C$; see also Equation (2.10). Convolution is defined by

$$
f * g(x)=\int_{G} f\left(x y^{-1}\right) g(y) d y
$$

for $f$ and $g$ smooth functions. By using the series of matrix coefficients (1.3) this convolution can be extended to $k * f$ for $k \in \mathcal{D}^{h}$ and $f \in C^{\infty}$.

In Section 3 we prove the result for the group $S U(2)$. This special case is essential since the proof of the main result is by reducing the general case to that of $S U(2)$. The main result is proved in Section 4 .

In Section 5 we give two examples. The first is that of the Laplacian operator. As well as being interesting in its own right it is necessary technically for the proof in Section 4. The other example is of the operator $L$ from [2]. This shows that we cannot strengthen the conclusion in general. While some operators, the Laplacian for example, are both convolution operators and classical differential operators, this operator, $L$, is only of type $\left(\frac{1}{2}, \frac{1}{2}\right)$ and cannot be of type $(\rho, 1-\rho)$ for $\rho>\frac{1}{2}$, see [2] for more details of this.

The author was supported by the C.C. Hsiung fund. 


\section{Convolution operators and smoothness.}

Let $G$ be a compact connected semisimple Lie group. Fix a maximal torus $T$ in $G$ and choose a set of positive roots $P$. Let $\rho$ be half the sum of the positive roots. Define the dimension polynomial by

$$
d(\lambda)=\prod_{\alpha>0}\langle\lambda, \alpha\rangle / \prod_{\alpha>0}\langle\rho, \alpha\rangle,
$$

where $\langle$,$\rangle is the negative of the Killing form and both products are taken$ over the positive roots. If $\pi_{\lambda}: G \rightarrow$ Aut $E_{\lambda}$ is the irreducible representation with highest weight $\lambda$ then

$$
\operatorname{dim} E_{\lambda}=d(\lambda+\rho) .
$$

Pick a basis $\left\{v_{i}\right\}$ for $E_{\lambda}$ then a basis for the matrix coefficients of $\pi_{\lambda}$ are the functions

$$
u_{\lambda}^{i j}(g)=\left\langle\pi_{\lambda}(g) v_{i}, v_{j}\right\rangle .
$$

For two functions, $f$ and $g$, on $G$ we define the inner product, $\langle$,$\rangle , and$ convolution, $*$, by

$$
\langle f, g\rangle=\int_{G} f(x) \overline{g(x)} d x,
$$

and

$$
f * g(x)=\int_{G} f\left(x y^{-1}\right) g(y) d y .
$$

In these we use the Haar measure which is normalized so that $G$ has volume 1, i.e.

$$
\int_{G} 1 d x=1 .
$$

We further pick the basis $\left\{v_{i}\right\}$ of $E_{\lambda}$ to be orthogonal with respect to an invariant inner product on $E_{\lambda}$; and this is scaled so that the inner product and convolution are:

$$
\begin{aligned}
\left\langle u_{\lambda}^{i j}, u_{\mu}^{k \ell}\right\rangle & = \begin{cases}d(\lambda+\rho)^{-1} & \text { if } i=k, j=\ell, \lambda=\mu \\
0 & \text { otherwise, }\end{cases} \\
u_{\lambda}^{i j} * u_{\mu}^{k \ell} & = \begin{cases}d(\lambda+\rho)^{-1} u_{\lambda}^{i j} & \text { if } i=k, j=\ell, \lambda=\mu \\
0 & \text { otherwise. }\end{cases}
\end{aligned}
$$


The Fourier coefficients of a function $f$ on $G$ are defined by

$$
\hat{f}_{\lambda}^{i j}=\left\langle f, u_{\lambda}^{i j}\right\rangle
$$

Thus if $f=\sum_{\lambda, i, j} a_{\lambda}^{i j} u_{\lambda}^{i j}$ then $a_{\lambda}^{i j}=d(\lambda+\rho) \hat{f}_{\lambda}^{i j}$. If $f \in L^{2}$ such a series always exists and converges in mean. This need not be true for $f \in L^{p}$ with $p \neq 2$, see [6]. However, if $f$ is suitably differentiable then even stronger results hold, see [7] for the following result.

Proposition 2.1. If $f \in C^{h}(G)$ with $h$ even and $h>\frac{1}{4} \operatorname{dim} G$ and if $f=\sum a_{\lambda}^{i j} u_{\lambda}^{i j}$ then $\left|a_{\lambda}^{i j}\right|=O\left(\|\lambda\|^{r}\right)$ with $r \leq-h-m$, where $m$ is the number of positive roots. Conversely if $\left|a_{\lambda}^{i j}\right|=O\left(\|\lambda\|^{r}\right)$ then $f \in C^{h}(G)$ for $h \leq-r-m$ providing $h$ even and $h>\frac{1}{4} \operatorname{dim} G$. Further the series $\sum a_{\lambda}^{i j} u_{\lambda}^{i j}$ converges absolutely and uniformly.

Remark. The result in [7] is stated in terms of the Fourier coefficients $\hat{f}_{\lambda}^{i j}$. Our statement follows since $a_{\lambda}^{i j}=d(\lambda+\rho) \hat{f}_{\lambda}^{i j}$ and $d(\lambda+\rho)=O\left(\|\lambda\|^{m}\right)$.

We define a space of distributions $\mathcal{D}^{h}$ :

$$
\mathcal{D}^{h}=\left\{f: f=\sum a_{\lambda}^{i j} u_{\lambda}^{i j} \quad \text { with } \quad a_{\lambda}^{i j}=O\left(|\lambda|^{h-\ell-m}\right)\right\}
$$

where $\ell=\operatorname{rank} G$ and $m$ is the number of positive roots. Then by Proposition 2.1 and the results of [7] we have that $\mathcal{D}^{h}$ is dual to $C^{h}(G)$ providing $h$ is even and $h>\frac{1}{4} \operatorname{dim} G$. The operators we are concerned with are $O P \mathcal{D}^{h}$ which are defined by $K \in O P \mathcal{D}^{h}$ if

$$
K f=k * f \quad \text { for } k \in \mathcal{D}^{h} .
$$

Clearly, from (2.8) and Proposition 2.1 if $f \in C^{\infty}(G)$ then $K f \in C^{\infty}(G)$.

\section{The case of $S U(2)$.}

The group $S U(2) \cong S^{3} \subset \mathbb{C}^{2}$. Let $(z, w)$ be coordinates for $\mathbb{C}^{2}$ then

$$
S U(2)=\left\{\left(\begin{array}{rr}
z & w \\
-\bar{w} & \bar{z}
\end{array}\right): z \bar{z}+w \bar{w}=1\right\},
$$

where - denotes complex conjugation. There is a natural action of $S U(2)$ on $\mathbb{C}^{2}$ :

$$
\left(\begin{array}{rr}
z & w \\
-\bar{w} & \bar{z}
\end{array}\right)\left(\begin{array}{l}
x \\
y
\end{array}\right)=\left(\begin{array}{r}
z x+w y \\
-\bar{w} x+\bar{z} y
\end{array}\right) .
$$


This extends to an action on $\mathcal{S}^{k}\left(\mathbb{C}^{2}\right)$, the symmetric $k$-fold tensor product of $\mathbb{C}^{2}$, which can be identified as the space of homogeneous polynomials of degree $k$ in two variables. These actions are in fact irreducible representations of $S U(2)$ and all irreducible representations are given in this way. To be consistent with the notation of Section 2, index the representations by $\lambda=\frac{1}{2} k$. Then the matrix coefficients of the irreducible representations are given by polynomials.

Proposition 3.1. The matrix coefficient $u_{\lambda}^{i j}$ is polynomial homogeneous of degree $2 \lambda$ in the four variables $z, \bar{z}, w$ and $\bar{w}$.

Let $M$ be a thickening of $S U(2)$ :

$$
M=\left\{(z, w) \in \mathbb{C}^{2}: \frac{1}{2}<z \bar{z}+w \bar{w}<2\right\} .
$$

Let $\nu$ be the 3 dimensional sub bundle of $T(M)$ which is orthogonal to the radial direction in $M$ when we regard $M \subset \mathbb{R}^{4}$. On $\mathbb{R}^{4}-\{0\}$ use polar coordinates $(r, \sigma)$ with $r>0$ and $\sigma \in \mathcal{S}^{3}$. Now set $s=r^{2}$ then the Euclidean dilation $t \cdot(r, \sigma)=(t r, \sigma)$ becomes a Heisenberg dilation $t \cdot(s, \sigma)=\left(t^{2} s, \sigma\right)$. Set $\tilde{u}_{\lambda}^{i j}=u_{\lambda}^{i j} / r^{4 \lambda}$. Then, since $u_{\lambda}^{i j}$ is given by a homogeneous polynomial, we have $\tilde{u}_{\lambda}^{i j}$ is homogeneous under the Heisenberg dilation:

$$
\tilde{u}_{\lambda}^{i j}(t \cdot(s, \sigma))=t^{-2 \lambda} \tilde{u}_{\lambda}^{i j}(s, \sigma) .
$$

If $k=\sum a_{\lambda}^{i j} u_{\lambda}^{i j} \in \mathcal{D}^{h}$ then define the operator $\tilde{K}$ by

$$
\tilde{K} f(r, s)=\int_{0}^{\infty} \int_{S U(2)} \tilde{k}\left(r s^{-1}, x y^{-1}\right) f(s, y) d y d s .
$$

Then if $f$ has support in $S U(2)$ we have

$$
\tilde{K} f(1, x)=K f(x),
$$

for $x \in S U(2)$.

An operator $Q$ of type $\left(\frac{1}{2}, \frac{1}{2}\right)$, that is $Q \in O P S_{\frac{1}{2}, \frac{1}{2}}(M)$, is one that has a symbol $q(x, \xi)$ which satisfies the following growth limit:

$$
\left|D_{x}^{\alpha} D_{\xi}^{\beta} q(x, \xi)\right|<C(1+|\xi|)^{n+\frac{1}{2}|\alpha|-\frac{1}{2}|\beta|}
$$

Here $\alpha$ and $\beta$ are multi-indices, the inequality holds for $x$ in a compact subset $K$ of $M$ and $C$ is a constant depending on $K, \alpha$ and $\beta$ but independent of $x$ and $\xi$. 
Proposition 3.2. $\tilde{K} \in O P S_{\frac{1}{2}, \frac{1}{2}}(M)$.

Proof. Since $\tilde{K}$ is convolution with $\tilde{k}=\sum a_{\lambda}^{i j} \tilde{u}_{\lambda}^{i j}$ and $\tilde{u}_{\lambda}^{i j}$ is homogeneous of degree $-2 \lambda$ under the Heisenberg dilation we have $\tilde{K}$ is a $\nu$-operator of degree $n$, for some $n$, as defined in [1]. Further, in [1], it is shown that such operators are of type $\left(\frac{1}{2}, \frac{1}{2}\right)$, which completes the proof.

Theorem 3.3. The operator $K$ is of type $\left(\frac{1}{2}, \frac{1}{2}\right)$.

Proof. From Equation (3.6) we have that $K$ is the restriction of the operator $\tilde{K}$ to functions having in $S U(2)$. By Proposition $3.2 \tilde{K}$ is of type $\left(\frac{1}{2}, \frac{1}{2}\right)$ and so has a symbol satisfying inequality (3.7). Thus by restriction $K$ has a symbol satisfying (3.7) and so is of type $\left(\frac{1}{2}, \frac{1}{2}\right)$.

\section{Reduction of the general case to $S U(2)$.}

On the compact semisimple Lie group $G$ let $K f=k * f$ where $k \in \mathcal{D}^{h}$ and let $\Delta$ be the Laplace. First we show how we may take $k$ to be a differentiable function.

Lemma 4.1. $K=\Delta^{\nu} K_{1}+A$, where $\nu=\frac{1}{2} h+\frac{1}{2} \operatorname{dim} G+\varepsilon$ with $\varepsilon=0$ or $\frac{1}{2}$ so that $\nu$ is an integer, $K_{1} f=k_{1} * f$ where $k_{1} \in C^{h_{1}}$ with $h_{1}=\operatorname{dim} G+\ell$ an even integer and $A f=a^{o} * f$, convolution by the constant term of $k$.

Proof. Define $k_{1}$ be the series

$$
k_{1}=\sum_{\lambda, i, j} c(\lambda)^{-\nu} a_{\lambda}^{i j} u_{\lambda}^{i j}
$$

where the term $\lambda=0$ is omitted and $c(\lambda)=\langle\lambda, \lambda+2 \rho\rangle$. Since the eigenvalues of the Laplace are:

$$
\Delta u_{\lambda}^{i j}=c(\lambda) u_{\lambda}^{i j}
$$

the formula of the proposition holds. Now

$$
c(\lambda)^{-\nu} a_{\lambda}^{i j}=O\left(|\lambda|^{h-2 \nu-\ell-m}\right),
$$

since $k \in \mathcal{D}^{h}$ gives $a_{\lambda}^{i j}=O\left(|\lambda|^{h-\ell-m}\right)$. Then by applying Proposition 2.1 we get the $k_{1} \in C^{h_{1}}$ and the lemma is proved.

Under the adjoint action the Lie algebra of $G$ splits as the sum

$$
\mathfrak{G}=\mathfrak{t} \underset{\alpha}{\oplus} V_{\alpha},
$$


where $\mathfrak{t}$ is the Lie algebra of the maximal tours, the sum is over the positive root $\alpha$ and each $V_{\alpha}$ is a 2 dimensional subspace of $\mathfrak{G}$. Fix a positive root $\alpha$ and let $h_{\alpha} \in \mathfrak{t}$ be the root vector corresponding to $\alpha$. Then $h_{\alpha} \mathbb{R} \oplus V_{\alpha}$ is a 3 dimensional subalgebra of $\mathfrak{G}$ isomorphic to $S U(2)$. By integration we get a homomorphism

$$
i_{\alpha}: S U(2) \rightarrow G
$$

which has a discrete kernel and whose derivative is the isomorphism from $S U(2)$ onto $h_{\alpha} \mathbb{R} \oplus V_{\alpha}$. Taking the product of these over all the positive roots gives a map

$$
i: S U(2) \times \cdots \times S U(2) \rightarrow G .
$$

Lemma 4.2. The map $i=\prod_{\alpha} i_{\alpha}$ of (4.6) is a surjective homomorphism.

Proof. Clearly $i$ is a homomorphism and the image $\prod$ of $i$ is a Lie subgroup of $G$. Since the derivative of $i$ is surjective the image of $i$ contains an open neighborhood of the identity in $G$. Since such a neighborhood generates $G$, $i$ must be surjective.

Theorem 4.3. Let $K$ be a convolution operator on $G$ then $K$ is of type $\left(\frac{1}{2}, \frac{1}{2}\right)$.

Proof. Let $K f=k * f$. Then by Lemma 4.1 we may suppose that $k$ is a differentiable function. If $k$ is a distribution we work with the differentiable function $k_{1}$ as defined in Lemma 4.1. We lift $K$ to $\prod_{\alpha} S U(2)$ by defining

$$
\tilde{k}=k \circ i
$$

and setting $\tilde{K} f=\tilde{k} * f$ with the convolution on the group $\prod_{\alpha} S U(2)$. Let $\tilde{k}_{\alpha}=\tilde{k} \mid S U(2)$ where we have chosen the copy of $S U(2)$ corresponding to the positive root $\alpha$. Thus we have

$$
\tilde{k}=\prod_{\alpha} \tilde{k}_{\alpha}
$$

Let $\tilde{K}_{\alpha}$ be the operator corresponding to convolution of $\tilde{k}_{\alpha}$ on the copy of $S U(2)$ corresponding to $\alpha$. Then we have

$$
\tilde{K}=\prod_{\alpha} \tilde{K}_{\alpha}
$$

By Theorem 3.3 each operator $\tilde{K}_{\alpha}$ is of type $\left(\frac{1}{2}, \frac{1}{2}\right)$. Thus $\tilde{K}$ is of type $\left(\frac{1}{2}, \frac{1}{2}\right)$ and hence so is $K$ which completes the proof of the theorem. 


\section{Two Examples and the order of an operator.}

Let $\Delta$ be the Laplacian on $G$. Then it is well known that the eigenfunctions of $\Delta$ are $u_{\lambda}^{i j}$ and the corresponding eigenvalues are given by

$$
\Delta u_{\lambda}^{i j}=c(\lambda) u_{\lambda}^{i j}, c(\lambda)=\langle\lambda, \lambda+2 \rho\rangle .
$$

Here $\rho$ is half the sum of the positive roots. Now we can form the distribution

$$
\Omega(x)=\sum_{\lambda, i, j} d(\lambda+\rho) c(\lambda) u_{\lambda}^{i j}
$$

and from (2.8) and (5.1) we obtain immediately the following result.

Lemma 5.1. The Laplacian is a convolution operator: $\Delta f=\Omega * f$. Further $\Omega \in \mathcal{D}^{h}$ with $h=\operatorname{dim} G+2$.

If $\nu$ is a positive integer $\Delta^{\nu} u_{\lambda}^{i j}=c(\lambda)^{\nu} u_{\lambda}^{i j}$ for all $\lambda$. However, if $\nu$ is a negative this only holds for $\lambda \neq 0$. In either case we have the formula $\Delta^{\nu} f=\Omega * f$ providing the sum in (5.2) is taken over nonzero $\lambda$, and $\Omega^{\nu}=$ $\sum_{\lambda, i, j} d(\lambda+\rho) c(\lambda)^{\nu} u_{\lambda}^{i j}$ with $\lambda \neq 0$.

The second example is the operator $L$ from [2]. This is defined in the following way. The matrix coefficients are given by

$$
u_{\lambda}^{i j}(x)=\left\langle\pi_{\lambda}(x) v_{i} v_{j}\right\rangle,
$$

where $\left\{v_{i}\right\}$ is an orthogonal basis for the representation space $E_{\lambda}$. Pick this basis so that $v_{1}$ is the highest weight vector for $\pi_{\lambda}$. Then if $f=\sum_{\lambda, i, j} a_{\lambda}^{i j} u_{\lambda}^{i j}$ we define $L$ by

$$
L f=\sum_{\lambda, j} a^{1 j \lambda} u^{1 i \lambda}
$$

that is $L$ is a projection onto the subspace spanned by matrix coefficients with highest weight vectors. As with the Laplacian the following is immediate. Define the distribution $\ell$ by

$$
\ell(x)=\sum_{\lambda, j} d(\lambda+\rho) u_{\lambda}^{1 j}
$$

Lemma 5.2. The operator $L$ is a convolution operator $L f=\ell * f$. Further $\ell \in \mathcal{D}^{h}$ with $h=\operatorname{dim} G$.

Remark. This distribution $\ell$ differs from that in [2] since we have used a different normalization for the volume $G$ and the matrix coefficients. 
It is straightforward to calculate the order of a convolution operator.

Theorem 5.3. Let $K$ be the operator given by convolution with $k \in \mathcal{D}^{h}$ : $K f=k * f$. Then $K$ has order $h-\operatorname{dim} G$.

Proof. First we suppose that $h-\operatorname{dim} G$ is even. Then let $f \in C^{r}$ where $r$ is even and both $r>\frac{1}{4} \operatorname{dim} G$ and $r>\frac{3}{4} \operatorname{dim} G+h$. Let $k=\sum a_{\lambda}^{i j} u_{\lambda}^{i j}$ and $f=\sum b_{\lambda}^{i j} u_{\lambda}^{i j}$. Then by Proposition 2.1 and (2.10)

$$
\begin{aligned}
& a_{\lambda}^{i j}=O\left(|\lambda|^{h-\ell-m}\right) \\
& b_{\lambda}^{i j}=O\left(|\lambda|^{-r-m}\right) .
\end{aligned}
$$

Now

$$
k * f=\sum d(\lambda+\rho)^{-1} a_{\lambda}^{i j} b_{\lambda}^{i j} u_{\lambda}^{i j}
$$

Since

$$
d(\lambda+\rho)^{-1} a_{\lambda}^{i j} b_{\lambda}^{i j}=O\left(|\lambda|^{h-r-\operatorname{dim} G-m}\right)
$$

and $h-\operatorname{dim} G$ is even, then by Proposition 2.1 we have

$$
k * f \in C^{r-h+\operatorname{dim} G} .
$$

This shows that the order of $K$ is $h-\operatorname{dim} G$ in the case that this is even.

In the case $h-\operatorname{dim} G$ is odd apply the above argument to $K^{2}$. Thus $K^{2}$ has order $2(h-\operatorname{dim} G)$ which is even. Hence $K$ has order $h-\operatorname{dim} G$ in the odd case.

\section{References}

[1] R. Beals and P. Greiner, Calculus in Heisenberg manifolds, Ann. of Math. Studies, 119 (1988), Princeton University Press, Princeton, NJ.

[2] H.D. Fegan, Second order operators with non-zero eta invariant, Canad. Math. Bull., 35(3) (1992), 341-353.

[3] P.B. Gilkey, The eta invariant of even order operators, Differential Geometry (F.J. Carreras, Om Medrano and A.M. Naviera, eds.), Lecture Notes in Math., 1410, Springer Verlag, Berlin, (1989), 202-211.

[4] L. Hörmander, Pseudodifferential operators and hypeoelliptic equation, Proc. Symp. Pure Math., 10 (1967), 138-183, Singular Integrals, American Math. Soc., Providence, RI.

[5] Y. Meyer, Wavelets and operators, Cambridge Studies in Advanced Mathematics, 37 (1992), Cambridge University Press. 
[6] R.J. Stanton and P.A. Thomas, Convergence of Fourier series on compact Lie groups, Bull. Amer. Math. Soc., 82 (1976), 61-62.

[7] M. Sugiura, Fourier series of smooth functions on compact Lie groups, Osaka J. Math., 8 (1971), 33-47.

[8] M.E. Taylor, Noncommunicative microlocal analysis, Part 1, Mem. of Amer. Math. Soc., 52(313) (1984).

Received October 31, 1994 and revised February 22, 1995.

\section{LEHIGH UNIVERSITY}

BETHLEHEM, PA 18015-3174

E-mail address: hdf3@lehigh.edu

Note: The Reference to Proposition 2.1 in the proof of Theorem 5.3 APPEARED AS "PROPOSITION 2.2" IN THE PRINTED VERSION. 